\title{
Patrones de regeneración en renovales de Drimys winteri en el centro-norte de la Isla de Chiloé: cambios de acuerdo al tamaño y la densidad relativa
}

\author{
Regeneration patterns for the Drimys winteri second-growth forests in north-central Chiloé \\ Island: changes according to size and relative density
}

\author{
Daniel P Soto $^{1 *}$, Pablo J Donoso ${ }^{1}$ \\ ${ }^{1}$ Universidad Austral de Chile, Instituto de Silvicultura, casilla 567, Valdivia, Chile. \\ *Autor de correspondencia: fono: 56-063-221742, fax: 56-063-221230, danielsoto@uach.cl
}

\begin{abstract}
SUMMARY
The present study aimed to register changes in regeneration density in Drimys winteri secondary forests in north-central Chiloé Island, according to size structure (dominant height, HT: $<16,16-19$ and $>19 \mathrm{~m}$ ), and quadratic mean diameter (QSD): $<12,12-$ 15 and $>15 \mathrm{~cm}$ ), relative density (RD: $<80$ and $>80 \%$ ), and slope (flat, 1-10 and 10-20). For this purpose 52 circular plots were sampled, each 200 to $400 \mathrm{~m}^{2}$ in size, on which the diameter of all trees $\geq 5 \mathrm{~cm}$, the height of five dominant trees, and the slope of the plot were measured. In each plot, four regeneration plots were sampled, where seedlings $(<2 \mathrm{~m}$ tall) and saplings $(\geq 2 \mathrm{~m}$ tall and $<5 \mathrm{~cm}$ in diameter) were tallied. Through analyses of variance and the least significant difference test we determined that: a) RD and slope did not have a significant effect on regeneration, b) regeneration of D. winteri and L. philippiana was significantly affected by size structure, both for saplings and seedlings, c) regeneration of Eucryphia cordifolia and Mirtaceae did not show significant differences according to size structure. Negative logarithmic regression models were obtained for $D$. winteri and E. cordifolia, and a power model was fitted for L. philippiana. Observed patterns of regeneration for regeneration density are consistent with the observed dominance of L. philippiana and Mirtaceae in old-growth forests of Chiloé.
\end{abstract}

Key words: stem exclusion, understory reinitiation, forest succession, secondary forests.

\section{RESUMEN}

Este estudio tuvo como objetivo evaluar los cambios de densidad de la regeneración en renovales de Drimys winteri en el centro norte de la Isla de Chiloé, de acuerdo a categorías de tamaño de los árboles (altura dominante: < 16, 16-19 y > 19 m; diámetro medio cuadrático: $<12,12-15$ y $>15 \mathrm{~cm}$ ), densidad relativa (DR: $<80$ y $>80 \%$ ) y pendiente plana, $<10$ y $10-20 \%$. En 52 parcelas, de $200 \mathrm{o} 400 \mathrm{~m}^{2}$, se registró diámetro de todos los árboles $\geq 5 \mathrm{~cm}$, altura de cinco árboles dominantes y pendiente del terreno. En cada parcela se muestrearon cuatro subparcelas de regeneración, donde se contabilizaron las plántulas $(<2$ m de altura) y los brinzales ( $\geq 2 \mathrm{~m}$ de altura y $<5 \mathrm{~cm}$ de diámetro). Mediante análisis de varianza y diferencias mínimas significativas se determinó que: a) la DR y la pendiente no tuvieron efectos significativos sobre la regeneración, b) la regeneración de $D$. winteri y Laureliopsis philippiana fue significativamente diferente según la estructura de tamaños, tanto para brinzales como para plántulas, pero c) aquellas de Eucryphia cordifolia y de las mirtáceas no fueron significativamente distintas según tamaño. Se ajustaron modelos de regresión del tipo logarítmico negativo para la densidad de regeneración de D. winteri y E. Cordifolia, y del tipo potencial para L. philippiana, con la altura del bosque como variable independiente. Los patrones observados en la densidad de la regeneración son consistentes con la dominancia de L. philippiana y mirtáceas observada en estadíos sucesionales avanzados en bosques de Chiloé.

Palabras clave: exclusión fustal, reinicio regeneración, sucesión forestal, bosques secundarios.

\section{INTRODUCCIÓN}

La sucesión forestal secundaria en bosques templados ha sido clasificada en cuatro etapas: iniciación, exclusión fustal, reinicio de la regeneración y estado adulto (Oliver 1981, Oliver y Larson 1996). Los renovales o bosques secundarios jóvenes corresponden a aquéllos de las dos primeras categorías. Este esquema asume que durante la exclusión fustal no se establece regeneración bajo el dosel de los árboles que dominan un rodal; la regeneración sólo vuelve a ser un proceso importante durante la etapa de reinicio de la regeneración, en rodales de edad intermedia, cuando árboles de tamaño relativamente grande al morir generan espacios de entrada de luz y liberan otros recursos por un tiempo tal que existe la posibilidad de que éstos sean capturados por la regeneración antes 
del cierre de copas del dosel. Este esquema de la sucesión forestal ha sido utilizado y aceptado reiteradamente en la literatura de ecología y dinámica forestal (Smith et al. 1997, Hunter 1999, Kozlowski 2002, Nyland 2002), y se ha planteado que para fomentar la regeneración en la etapa de exclusión forestal se requiere manipular los rodales a través de raleos que afecten a distintos doseles del rodal.

A pesar del esquema señalado, también se ha observado que la duración de la etapa de exclusión fustal puede ser muy variable o inexistente para ciertas poblaciones (Palik y Pregitzer 1993). Si bien es relevante aplicar silvicultura en rodales jóvenes bajo alta competencia, por ejemplo a través de raleos, con el fin de disminuir la competencia entre los árboles, es poco frecuente que éstos se realicen con el objetivo de fomentar la regeneración en esta etapa de desarrollo, a pesar de que una regeneración temprana bajo el dosel del rodal permitiría establecer esquemas de silvicultura de especies mixtas, bietánea o multietánea. El tipo, intensidad y oportunidad de un raleo en esta etapa puede verse modificado de acuerdo a la existencia y magnitud de la regeneración avanzada. La o las intervenciones aplicadas, o la no realización de ellas, determinarán la composición, estructura y crecimiento del rodal.

La regeneración en rodales secundarios puede estar fuertemente influenciada por la existencia de fuentes de semilla cercanas a éstos (Palik y Pregitzer 1993), o por la existencia de claros, tipo de sustrato y características ambientales locales (Figueroa y Hernández 2001, Figueroa y Lusk 2001, Saldaña y Lusk 2003), pero también por la densidad y tamaño del rodal. Una densidad menor en un rodal permite mayor entrada de luz y menor competencia. Densidades relativas (sensu Reineke 1933, Curtis 1970) altas (e.g. < 80\%) son un reflejo de fuerte competencia y alta mortalidad, además de afectar negativamente el crecimiento en diámetro y, en casos extremos, también el crecimiento en altura (Curtis 1970, Drew y Fleweling 1977, Puettmann et al. 1992, 1993, Zeide 1995, Nyland 2002, Long et al. 2004). En renovales, además, las densidades altas deberían ser una limitante para el establecimiento de la regeneración. Similarmente, renovales de mayor tamaño (diámetro medio o altura), para un mismo sitio, reflejan una mayor edad. En ellos hay más posibilidades de creación de claros, y debieran estar más estratificados que rodales más jóvenes, todo lo cual facilitaría el establecimiento de la regeneración.

Para evaluar los efectos de la densidad y el tamaño de rodales sobre la regeneración en bosques secundarios, en este trabajo se analizaron bosques de Drimys winteri (J. R. et Forster) del centro norte de la Isla de Chiloé. Estos bosques constituyen una superficie forestal de gran envergadura del sur de Chile y en particular en la Isla de Chiloé (230 mil hectáreas, de las cuales el 34\% se presenta en la Isla de Chiloé; CONAF et al. 1997, Navarro et al. 1999); dominan el paisaje forestal de esta región, presentan una alta productividad (Corvalán et al. 1987, Navarro et al. 1999), y madera de muy buenas propiedades (Rodríguez 1997). La magnitud de la regeneración en distintos estados de desarrollo de los renovales, y bajo distintos grados de densidad relativa, es una información relevante para planificar actividades silvícolas y para predecir la composición futura de estos bosques.

Este estudio tuvo como objetivo determinar los efectos de la estructura y la densidad sobre la regeneración arbórea de las principales especies en renovales de D. winteri del centro-norte de la Isla de Chiloé. Para ello se planteó a) evaluar los efectos de la densidad relativa sobre la abundancia y composición de la regeneración avanzada de las principales especies arbóreas en renovales de $D$. winteri, b) evaluar los cambios en la regeneración según la estructura de tamaños (altura dominante y diámetro medio) de los renovales y c) generar modelos de regeneración en función del tamaño de los renovales. La hipótesis de trabajo es: $D$. winteri posee escasa o nula regeneración bajo su propio dosel en renovales de esta especie, independientemente de la estructura o la densidad, pero la regeneración de especies más tolerantes, particularmente Laureliopsis philippiana (Looser) Schodde, aumenta en estadios más avanzados de los renovales, es decir, cuando éstos tienen mayor altura y diámetro, y en condiciones de menos densidad.

\section{MÉTODOS}

Área de estudio. La información se tomó en cuatro sectores de la región centro-norte de la Isla de Chiloé: Ancud, Chaquihual, Butalcura y Las Quemas (figura 1). Todos estos sectores se encuentran entre los 50 y $120 \mathrm{~m}$ de altitud, en el sector interior de Chiloé, es decir, al oriente de la Cordillera de la Costa. El clima es templado húmedo con fuerte influencia oceánica (Di Castri y Hajek 1976), la precipitación media anual es de $2.000 \mathrm{~mm}$ y la temperatura media anual de $12^{\circ} \mathrm{C}$. La temperatura media del mes más caluroso es $16^{\circ} \mathrm{C}$ (enero) y $5^{\circ} \mathrm{C}$ para el mes más frío (Aravena et al. 2002).

El origen de los suelos es de morrenas pleistocénicas con depósitos fluvioglaciales (Denton et al. 1999, Carmona et al. 2002). Los suelos presentan drenaje restringido la mayor parte del año, debido a la presencia de una línea impermeable de hierro y manganeso precipitado ante la presencia de oxígeno (depósito fluvioglacial) (Grez y Carmona 1982). Comúnmente, estos suelos son llamados ñadis y clasificados como Gleysoles (FAO-UNESCO 1971). Las características principales de estos suelos son su escasa profundidad $(<1 \mathrm{~m})$, fuerte acidez y saturación de humedad la mayor parte del año (Grez y Carmona 1982, Donoso et al. 1984).

Muestreo y toma de datos. Se muestrearon 52 parcelas de un tamaño de $200 \mathrm{~m}^{2}$ cuando estimativamente el diámetro 


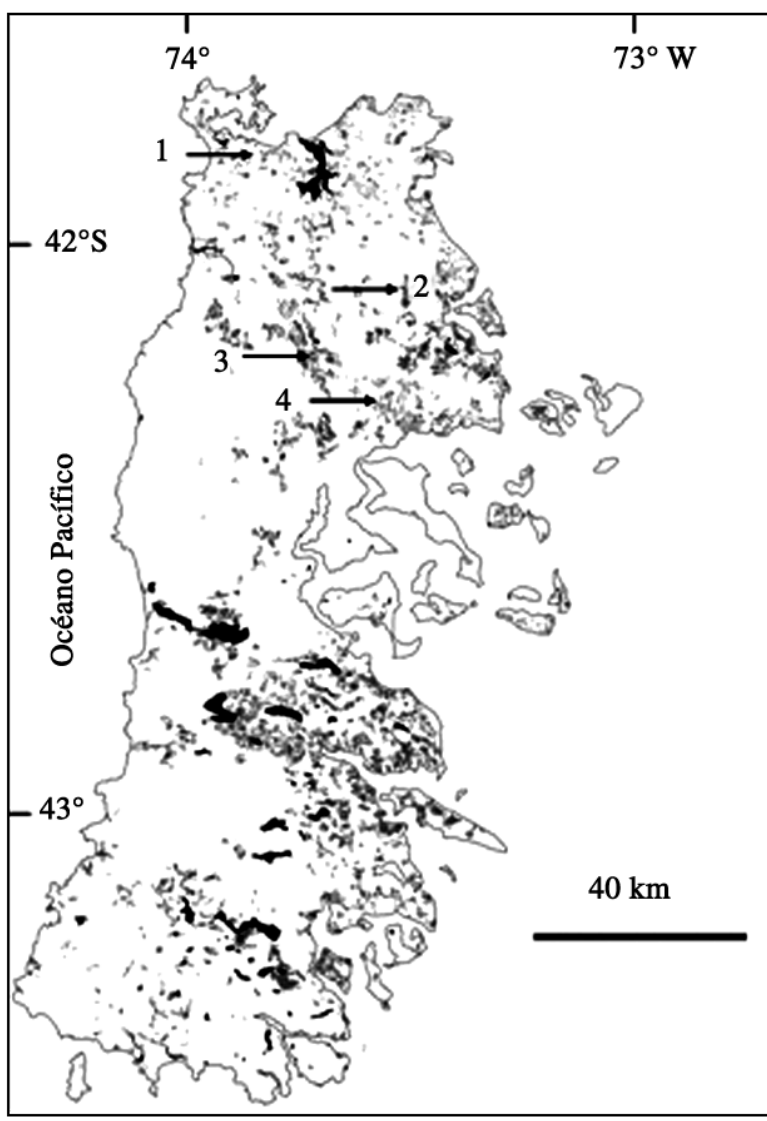

Figura 1. Localización de la zona de estudio en la Isla de Chiloé. En gris se muestran los renovales de Drimys winteri y en negro los cuerpos de agua y estuarios. 1: Ancud (Lechagua), 2: Chaquihual, 3: Butalcura y 4: Las Quemas.

Location of study sites in Chiloé Island.

Drimys winteri secondary forests in gray, lakes and estuaries in black. 1: Ancud (Lechagua), 2: Chaquihual, 3: Butalcura and 4: Las Quemas.

medio cuadrático (DMC) era $\leq 12 \mathrm{~cm}$, y de $400 \mathrm{~m}^{2}$ cuando éste se estimó superior a $12 \mathrm{~cm}$. Se realizó un muestreo estratificado a posteriori con distribución aleatoria de las parcelas. En cada una se registraron las especies arbóreas $\geq 5 \mathrm{~cm}$ de diámetro a la altura del pecho (DAP). A cinco árboles dominantes por parcela se les estimó la altura (AT) con un hipsómetro de ultrasonido VERTEX Haflog®, y la edad según técnicas dendrocronológicas (Stokes y Smile 1968; cuadro 1). La regeneración se registró en cuatro subparcelas cuadradas de $4 \mathrm{~m}^{2}$ en dirección a los puntos cardinales desde el centro de la parcela (a $4 \mathrm{~m}$ en parcelas de $200 \mathrm{~m}^{2}$ y a $6 \mathrm{~m}$ en parcelas de 400 $\mathrm{m}^{2}$ ), donde se registró el número de individuos de especies arbóreas: plántulas (individuos $<200 \mathrm{~cm}$ de altura) y brinzales (individuos $>2 \mathrm{~m}$ altura $\mathrm{y}<5 \mathrm{~cm}$ de DAP). En total se establecieron 208 parcelas de regeneración, las que corresponden a 52 conglomerados de 4 parcelas cada una (cuadro 1). Además, en cada parcela se registró la exposición y la pendiente.

Procesamiento y análisis de los datos. Los sitios se ordenaron según regeneración (plántulas y brinzales) promedio por conglomerado y, para el análisis, se eligieron las especies que se encontraron más comúnmente, vale decir, D. winteri, L. philippiana, Eucryphia cordifolia Cav. y mirtáceas [en conjunto: Myrceugenia planipes (Hooker et Arnott) Berg.; Myrceugenia parvifolia (DC) Kausel; Amomyrtus meli (Phil.) D. Legrand y Kausel; Amomyrtus luma (Mol.) Legrand y Kausel; y Luma apliculata (DC) Burret] (cuadro 2). Se definieron clases de DMC: $<12 \mathrm{~cm}$ $(\mathrm{n}=14$, rango $9,5-11,9 \mathrm{~cm}), 12-15 \mathrm{~cm}(\mathrm{n}=24$, rango $12-14,9 \mathrm{~cm})$ y $>15 \mathrm{~cm}(\mathrm{n}=14$, rango $15,2-19,7 \mathrm{~cm})$; de AT: $<16 \mathrm{~m}(\mathrm{n}=17$, rango $12,4-15,8 \mathrm{~m}), 16-19 \mathrm{~m}$ $(\mathrm{n}=25$, rango $16,0-18,9 \mathrm{~m})$ y $>19 \mathrm{~m}(\mathrm{n}=10$, rango $19,5-23,7 \mathrm{~m})$; y de pendiente: plana $=0 \%(\mathrm{n}=23)$, suave $1-<10 \%(\mathrm{n}=18)$ y mediana $10-20 \%(\mathrm{n}=11)$. Las parcelas se dividieron en dos categorías de densidad relativa: $<80 \%(\mathrm{n}=14$, rango $53-79 \%)$ y $>80 \%(\mathrm{n}=38$, rango 81-121\%), calculadas por Donoso et al. (2007).

Se realizó un análisis de varianza (ANDEVA) de un factor para la comparación de las densidades relativas, y uno de dos factores (categoría de tamaño [DMC o AT] y pendiente del terreno), con tres niveles para cada factor. Todas las variables independientes utilizadas fueron sometidas a la prueba de Levene para evaluar la homogeneidad de varianzas. En caso de un ANDEVA significativo, las separaciones de medias se efectuaron mediante la prueba de diferencias mínimas significativas (LSD en

Cuadro 1. Características dendrométricas generales de los rodales estudiados (media \pm desviación estándar).

General dendrometric characteristics of sampled stands (mean $\pm \mathrm{SD}$ ).

\begin{tabular}{lcccc}
\hline & Ancud & Butalcura & Chaquihual & Las Quemas \\
\hline Densidad (árboles/ha) & $7.179 \pm 2.122$ & $5.198 \pm 1.280$ & $4.749 \pm 2.090$ & $3.790 \pm 775$ \\
DMC (cm) & $11,4 \pm 1,9$ & $13,1 \pm 2,3$ & $14,8 \pm 2,9$ & $13,9 \pm 2,6$ \\
AT (m) & $17,1 \pm 2,5$ & $16,8 \pm 3,9$ & $18,6 \pm 3,2$ & $17,5 \pm 2,9$ \\
DR \% & $98,6 \pm 11,1$ & $82,2 \pm 9,5$ & $88,4 \pm 8,5$ & $80,9 \pm 10,3$ \\
Rango de edades (años) & $11-35$ & $26-44$ & $23-46$ & $21-46$ \\
$\mathrm{~N}^{\circ}$ conglomerados & 11 & 12 & 19 & 10 \\
$\mathrm{~N}^{\circ}$ parcelas regeneración & 44 & 48 & 76 & 40 \\
\hline
\end{tabular}


Cuadro 2. Regeneración de las principales especies arbóreas, segregadas en plántulas y brinzales (media \pm desviación estándar), en los sitios de estudio.

Regeneration of the main tree species for seedlings and saplings (mean $\pm \mathrm{SD})$.

\begin{tabular}{lcccccccc}
\hline \multirow{2}{*}{ Especie } & \multicolumn{2}{c}{ Ancud } & \multicolumn{2}{c}{ Butalcura } & \multicolumn{2}{c}{ Chaquihual } & \multicolumn{2}{c}{ Las Quemas } \\
\cline { 2 - 9 } & Plántulas & Brinzales & Plántulas & Brinzales & Plántulas & Brinzales & Plántulas & Brinzales \\
\hline Drimys winteri & $5.568 \pm 2.243$ & $3.579 \pm 1.621$ & $8.072 \pm 3.923$ & $3.958 \pm 2.287$ & $2.664 \pm 960$ & $2.269 \pm 804$ & $7.291 \pm 3.051$ & $2083 \pm 1062$ \\
Laureliopsis philippiana & $1.420 \pm 506$ & $397 \pm 270$ & $4.895 \pm 2.251$ & $1.458 \pm 463$ & $23.598 \pm 5.413$ & $3.865 \pm 886$ & $2.875 \pm 1.627$ & 0 \\
Eucryphia cordifolia & $5.192 \pm 3.556$ & $1.490 \pm 950$ & $5.138 \pm 2.956$ & $1.736 \pm 1.046$ & $1.022 \pm 530$ & 0 & $1.312 \pm 661$ & $1.687 \pm 533$ \\
Mirtáceas & $23.409 \pm 3.818$ & $10.340 \pm 2.682$ & $35.677 \pm 8.508$ & $11.093 \pm 3.828$ & $35.789 \pm 7.760$ & $10.756 \pm 3.447$ & $57.375 \pm 13.731$ & $15.750 \pm 4579$ \\
\hline
\end{tabular}

inglés). La significancia de las pruebas estuvo determinada por un valor de probabilidad $P<0,05$ (Sokal y Rohlf 1995).

Se ajustaron modelos de regresión para la regeneración. Cuando hubo diferencias significativas, según densidades relativas, se desarrollaron dos modelos; en caso contrario, se ajustó un solo modelo. Los modelos seleccionados fueron aquéllos con un mayor coeficiente de determinación $\left(\mathrm{R}^{2}\right)$, menor error estándar (EE) y menor probabilidad $(P)$ de cometer un error Tipo I. Para la selección del mejor modelo se ajustaron modelos del tipo lineal, logarítmico, exponencial, polinomial y potencial en el programa SPSS versión 11.5.

\section{RESULTADOS}

Regeneración de acuerdo a categorías de densidad relativa. Drimys winteri presentó mayor densidad de plántulas en la clase menor de DR $(P=0,050)$ (cuadro 3$)$ y a nivel de brinzales no hubo diferencias significativas $(P=0,289)$. Laureliopsis philippiana no presentó diferencias significativas para plántulas $(P=0,183)$ ni brinzales $(P=0,062)$, aunque para esta última categoría los resultados sugieren que mayores densidades relativas fa- vorecen la regeneración de esta especie (cuadro 3). Tampoco hubo diferencias significativas a nivel de plántulas $(P=0,976)$ ni brinzales $(P=0,264)$ para $E$. cordifolia. Finalmente, para las mirtáceas, al igual que para las otras especies analizadas, no hubo diferencias significativas según clase de DR tanto a nivel de plántulas $(P=0,567)$ como de brinzales $(P=0,760)$.

Densidad de la regeneración de acuerdo a categorías de diámetro, altura y pendiente del terreno. La pendiente no influyó significativamente en la densidad de plántulas en ninguna de las especies analizadas (cuadro 4). Drimys winteri tuvo mayor cantidad de plántulas en categorías de menor tamaño de AT $(P=0,0057$; figura 2$)$ y DMC $(P=0,0283$; figura 3$)$. La densidad de plántulas de L. philippiana fue significativamente distinta para las clases de AT $(P=0,0023)$ y DMC $(P<0,0001)$, concentrándose en mayor cantidad en clases de mayor de tamaño (cuadro 4, figuras 2 y 3). La densidad en E. cordifolia no fue significativamente distinta según clases de AT ( $P=0,2344$, figura 2$)$ y DMC $(P=0,0394$, figura 3$)$, pero sí lo fue según clases de DMC; en ambos casos la regeneración fue más abundante en las clases menores (figuras 2 y 3). Las mirtáceas no presentaron diferencias significativas para las clases de AT $(P=0,2082)$ y $\operatorname{DMC}(P=0,8042)$.

Cuadro 3. Regeneración de especies arbóreas (media \pm desviación estándar) segregadas según parcelas con $<80$ y $>80 \%$ de densidad relativa.

Regeneration of the main tree species (mean $\pm \mathrm{SD}$ ) for plots with $<80$ and $>80 \%$ relative density.

\begin{tabular}{lcccc}
\hline \multirow{2}{*}{ Especie } & \multicolumn{2}{c}{ Plántulas } & \multicolumn{2}{c}{ Brinzales } \\
\cline { 2 - 5 } & $<80$ & $>80$ & $<80$ & $>80$ \\
\hline Drimys winteri & $9.471 \pm 3.303$ & $3.980 \pm 1.143$ & $4.231 \pm 2.120$ & $2.467 \pm 644$ \\
Laureliopsis philippiana & $3.241 \pm 1.420$ & $9.984 \pm 2.980$ & $446 \pm 249$ & $2.088 \pm 510$ \\
Eucryphia cordifolia & $3.177 \pm 1.526$ & $3.088 \pm 1.368$ & $446 \pm 249$ & $1.354 \pm 315$ \\
Mirtáceas & $41.563 \pm 10.941$ & $36.280 \pm 5.052$ & $12.837 \pm 3.807$ & $11.513 \pm 2.159$ \\
\hline
\end{tabular}


Densidad de brinzales según categorías de diámetro, altura y pendiente del terreno. La densidad de brinzales de $D$. winteri fue significativamente diferente para las categorías de AT ( $P=0,0057$; figura 2 , cuadro 4$)$ y DMC ( $P=0,0111$; figura 3 , cuadro 4$)$. De igual manera, para $L$. philippiana se presentaron diferencias significativas para las clases de AT $(P=0,0194)$ y DMC $(P=$ $0,0051)$. La densidad de brinzales de E. cordifolia no tuvo diferencias significativas según clases de AT $(P=$ $0,5561)$ y DMC $(P=0,3532)$. La densidad de brinzales de mirtáceas presentó diferencias significativas según clases de AT $(P=0,0156)$ y DMC $(P=0,0023)$ (cuadro 4 , figuras 2 y 3$)$. La pendiente no fue un factor significa-

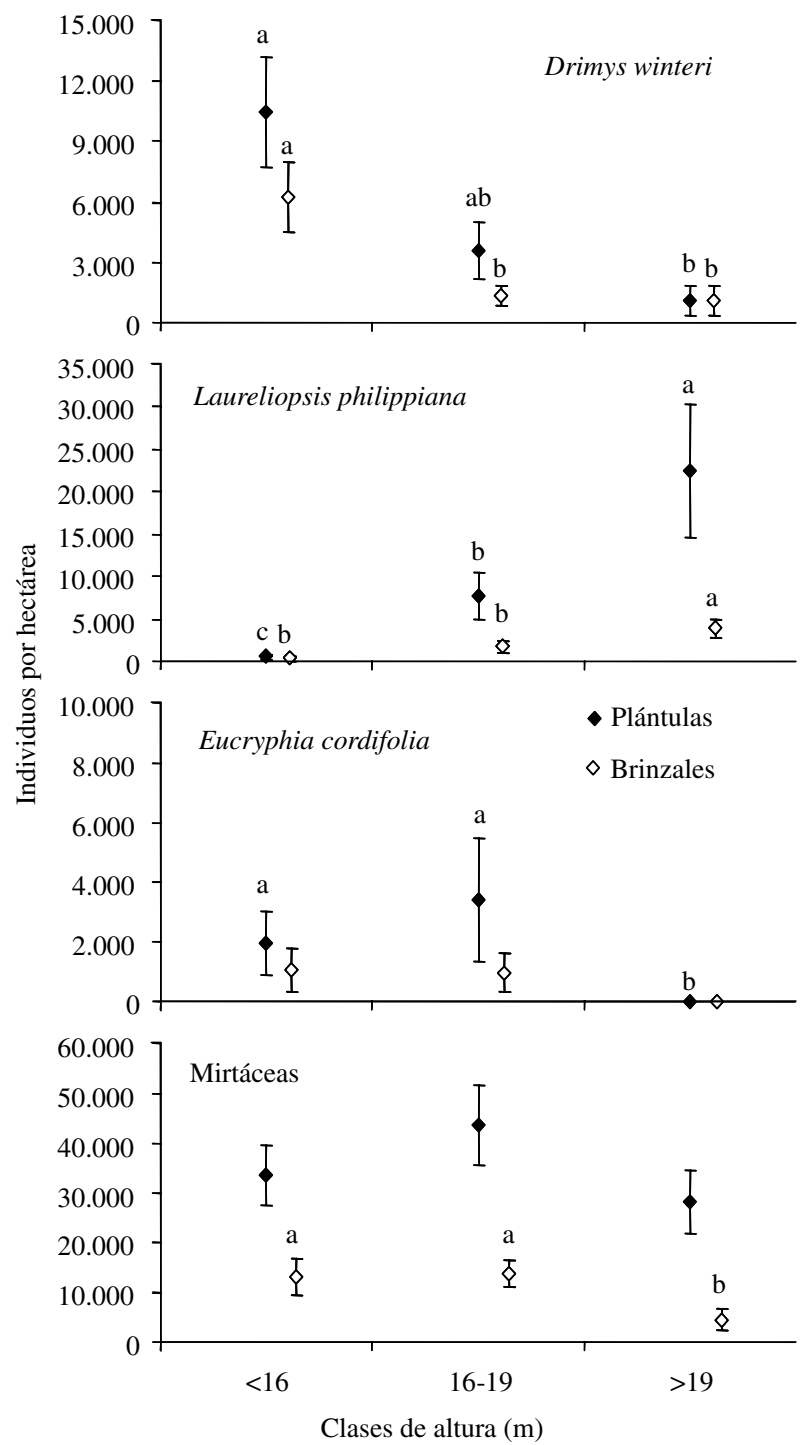

Figura 2. Regeneración (media \pm desviación estándar) según clase de altura en los sitios de estudio de la Isla de Chiloé. Letras diferentes muestran diferencias significativas en la prueba LSD $(P<0,05)$ entre clases de altura para plántulas y brinzales.

Regeneration (mean \pm SD) according to height class (AT) in study sites of Chiloé Island. Different letters show significant differences according to the LSD test $(P<0.05)$. tivo para ninguna de las especies; tampoco fue significativa la interacción de pendiente y categoría de tamaño.

Modelos de regeneración. La regeneración (plántulas y brinzales incluidos) de $D$. winteri se ajustó de manera logarítmica negativa (cuadro 5), siendo la AT mejor modelador de la regeneración total $(P=0,003)$. La regeneración de L. philippiana presentó un ajuste potencial
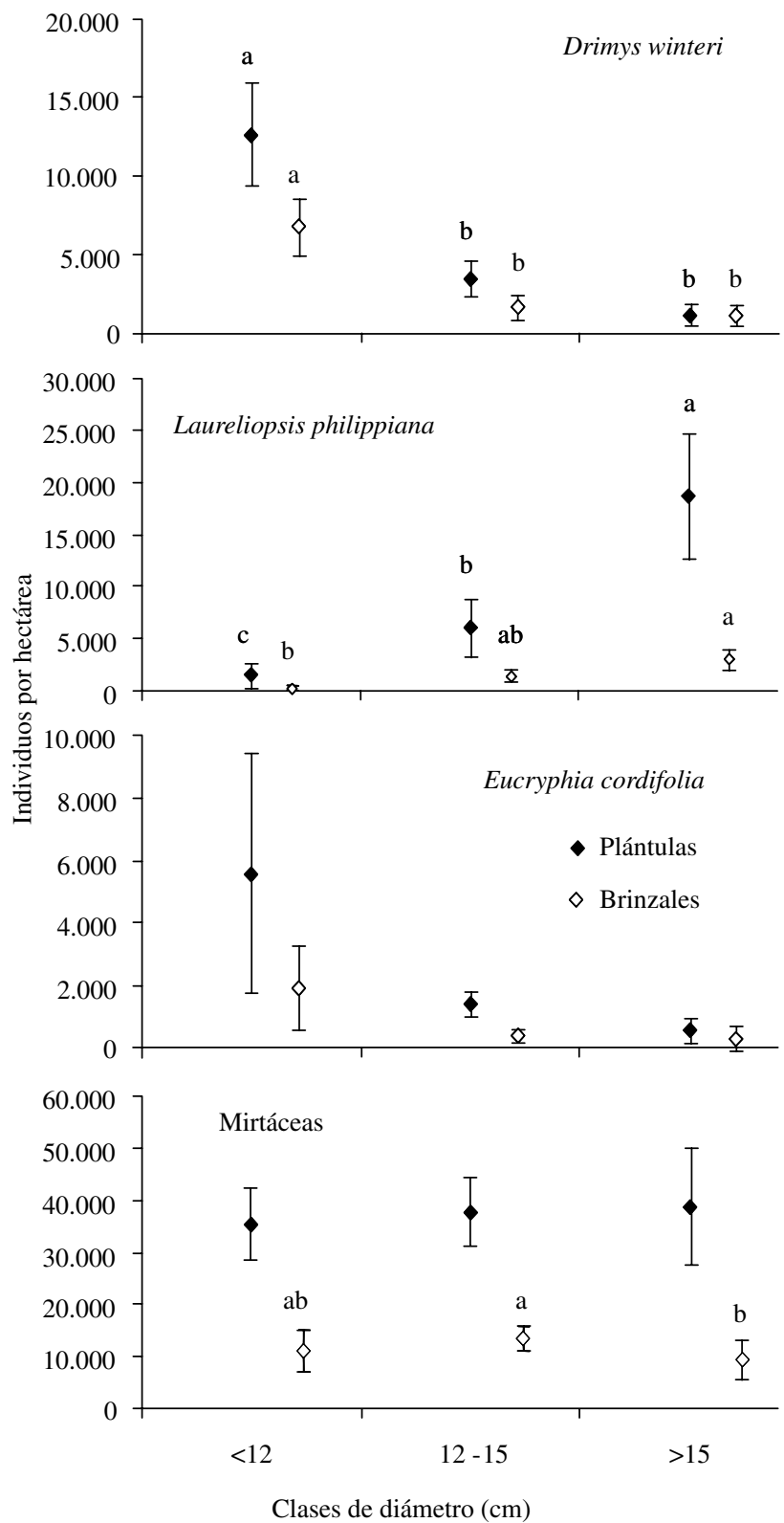

Figura 3. Regeneración (media \pm error estándar) según clase de diámetro DMC en los sitios de estudio de la Isla de Chiloé. Letras diferentes muestran diferencias significativas en la prueba LSD $(P<0,05)$ entre clases de diámetro para plántulas y brinzales.

Regeneration (mean $\pm \mathrm{SD}$ ) according to diameter class (DMC) in study sites of Chiloé Island. Different letters show significant differences according to the LSD test $(P<0.05)$. 
Cuadro 4. Resumen del análisis de varianza, valores F y significancia de los factores categoría de tamaños (CT), pendiente del terreno y su interacción.

Summary of analysis of variance, showing $\mathrm{F}$ values for CT (size category), slope, and interaction ( $\mathrm{CT}^{*}$ slope) for each main tree species.

\begin{tabular}{|c|c|c|c|c|c|}
\hline \multirow{2}{*}{ Especie } & \multirow{2}{*}{ Factor } & \multicolumn{2}{|c|}{ Plántulas } & \multicolumn{2}{|c|}{ Brinzales } \\
\hline & & DMC & AT & $\mathrm{DMC}$ & AT \\
\hline \multirow{3}{*}{$\begin{array}{l}\text { Drimys } \\
\text { winteri }\end{array}$} & $\mathrm{CT}$ & $3,86^{*}$ & $5,82 * *$ & $4,99 *$ & $5,82 * *$ \\
\hline & pendiente & $0,52 \mathrm{~ns}$ & $0,23 \mathrm{~ns}$ & $0,38 \mathrm{~ns}$ & $0,26 \mathrm{~ns}$ \\
\hline & CT x pendiente & $0,38 \mathrm{~ns}$ & $0,65 \mathrm{~ns}$ & $0,52 \mathrm{~ns}$ & $0,51 \mathrm{~ns}$ \\
\hline \multirow{3}{*}{$\begin{array}{l}\text { Laureliopsis } \\
\text { philippiana }\end{array}$} & CT & $14,91 * * *$ & $6,96^{* *}$ & $5,94 * *$ & $4,30 *$ \\
\hline & pendiente & $0,50 \mathrm{~ns}$ & $0,51 \mathrm{~ns}$ & $2,00 \mathrm{~ns}$ & $1,50 \mathrm{~ns}$ \\
\hline & CT x pendiente & $1,10 \mathrm{~ns}$ & $1,69 \mathrm{~ns}$ & $0,53 \mathrm{~ns}$ & $2,65 \mathrm{~ns}$ \\
\hline \multirow{3}{*}{$\begin{array}{l}\text { Eucryphia } \\
\text { cordifolia }\end{array}$} & $\mathrm{CT}$ & $3,47 *$ & $1,50 \mathrm{~ns}$ & $1,06 \mathrm{~ns}$ & $0,59 \mathrm{~ns}$ \\
\hline & pendiente & $0,17 \mathrm{~ns}$ & $0,05 \mathrm{~ns}$ & $0,07 \mathrm{~ns}$ & $0,01 \mathrm{~ns}$ \\
\hline & CT x pendiente & $0,29 \mathrm{~ns}$ & $0,88 \mathrm{~ns}$ & $0,04 \mathrm{~ns}$ & $1,02 \mathrm{~ns}$ \\
\hline \multirow[t]{3}{*}{ Mirtáceas } & $\mathrm{CT}$ & $0,22 \mathrm{~ns}$ & $1,62 \mathrm{~ns}$ & $6,93 * *$ & $4,56^{*}$ \\
\hline & pendiente & $0,49 \mathrm{~ns}$ & $0,02 \mathrm{~ns}$ & $0,56 \mathrm{~ns}$ & $0,10 \mathrm{~ns}$ \\
\hline & CT $x$ pendiente & $0,94 \mathrm{~ns}$ & $4,03^{*}$ & $0,82 \mathrm{~ns}$ & $0,94 \mathrm{~ns}$ \\
\hline
\end{tabular}

ns: no significativo, $* P<0,05, * * P<0,01, * * * P<0,001$.

Cuadro 5. Modelos de regeneración total (densidad de plántulas y brinzales por hectárea) según diámetro medio cuadrático (DMC) y altura dominante (AT) en renovales de Drimys winteri en la Isla de Chiloé $(\mathrm{n}=52)$.

Regeneration models (including seedlings and saplings) according to quadratic stand diameter (DMC in Spanish) and dominant height (AT in Spanish) in D. winteri secondary forests in Chiloé Island $(\mathrm{n}=52)$.

\begin{tabular}{lllll}
\hline \multirow{2}{*}{ Especies } & \multirow{2}{*}{ Modelos } & \multicolumn{3}{c}{ Estimadores } \\
\cline { 3 - 5 } & & $\mathrm{R}^{2}$ & $\mathrm{EE}$ & $\mathrm{F}$ \\
\hline \multirow{2}{*}{ Drimys winteri } & $62.120,4-21.547,4 \mathrm{Ln}(\mathrm{DMC})$ & 0,837 & $2.369,5$ & $37,70^{* *}$ \\
& $80.005,1-26.421,1 \mathrm{Ln}(\mathrm{AT})$ & 0,959 & $1.201,6$ & $69,44^{* *}$ \\
& & & & \\
Laureliopsis & $2,52 \times 10^{-6}(\mathrm{DMC})^{7,55436}$ & 0,944 & 0,393 & $51,22^{* *}$ \\
philippiana & $3,09 \times 10^{-8}(\mathrm{AT})^{9,0006}$ & 0,968 & 0,353 & $93,33^{* *}$ \\
& & & & \\
Eucryphia & $23.845,9-7.952,8 \mathrm{Ln}(\mathrm{DMC})$ & 0,884 & 718,5 & $22,85^{*}$ \\
cordifolia & $22.220,8-1.204,5 \mathrm{Ln}(\mathrm{AT})$ & 0,895 & $1.538,9$ & $24,5^{* *}$ \\
\hline
\end{tabular}

ns: no significativo, $* P<0,05, * * P<0,01$.

positivo, siendo la AT $(P=0,002)$ mejor estimador que el DMC $(P=0,005)$. En E. cordifolia el mejor ajuste fue logarítmico negativo para la regeneración $(P=0,01)$, siendo mejor estimador la AT (cuadro 5). La regeneración de mirtáceas no presentó ajustes significativos para ningún modelo.

\section{DISCUSIÓN}

Las densidades de plántulas encontradas en los renovales del centro norte de la Isla de Chiloé (entre 23 y 57 mil plántulas por hectárea) son similares a aquéllas reportadas por Appel (1993) para renovales en la Cordillera de la Costa de Valdivia (28 mil plántulas/ha en promedio), y por Loewe (1987) en la mayoría de los sitios que estudió en las provincias de Llanquihue y Chiloé (entre 20 y 47 mil plántulas/ha en ocho localidades, sobre 100 mil plántulas/ha en otras dos localidades). También los valores son similares a aquéllos reportados por Aravena (1991) y Aravena et al. (2002) en bosques sucesionales jóvenes (renovales de $D$. winteri con mirtáceas) en la parte norte de la Isla de Chiloé, es decir, del orden de 30 a 50 mil plántulas y de 3 a 10 mil brinzales por hectárea. Sin embargo, estos valores promedio poco ilustran acerca del futuro de estas plántulas, ya que algunas (las de especies más intolerantes a la sombra) podrían ser sólo efímeras, mientras que otras (las de especies más tolerantes) podrían tener mayores posibilidades de desarrollo.

Efectos de la densidad relativa sobre la regeneración. La densidad relativa (DR) sólo resultó ser marginalmente un factor explicativo de la densidad de regeneración en los renovales analizados (mayor regeneración de $D$. winteri y L. philippiana en renovales menos densos y más densos, respectivamente; cuadro 3). Este resultado puede obedecer a que, aunque se usó una categoría de baja y otra de alta densidad relativa, la primera incluyó renovales con densidades en promedio cercanas a $70 \%$, lo que representa una condición de densidad relativamente alta, con fuerte competencia y doseles cerrados (sensu Nyland 2002, Long et al. 2004). En renovales con menores densidades relativas, que es difícil hallar en renovales no manejados, es probable encontrar densidades de regeneración significativamente mayores. Por ejemplo, Appel (1993) determinó valores significativamente mayores de regeneración en renovales de $D$. winteri sometidos a raleos en la ladera occidental de la Cordillera de la Costa de Valdivia, cuya densidad relativa fue del orden de 40 a $50 \%$. Con densidades bajas hay aumento de luminosidad hacia el dosel inferior y aumentan la temperatura y la descomposición de la materia orgánica, condiciones con las cuales mejoran las posibilidades de regeneración de especies más intolerantes a la sombra, tales como E. Cordifolia y D. winteri (Donoso 1993, Weinberger 1997, Figueroa y Hernández 2001, Saldaña y Lusk 2003).

Regeneración según el tamaño medio de renovales. Los patrones generales de regeneración observados fueron distintos para cada una de las tres especies principales evaluadas y para las mirtáceas. Drimys winteri disminuyó su regeneración con aumentos en el tamaño medio de los renovales. Contrariamente, L. philippiana aumentó su regeneración en renovales de mayor tamaño medio; 
E. cordifolia tuvo mayores densidades de plántulas en renovales de altura intermedia, pero tanto para plántulas como para brinzales la densidad disminuyó en renovales de mayor diámetro. La densidad de mirtáceas fue independiente del tamaño de los renovales.

La mayor abundancia de plántulas de $D$. winteri en renovales más delgados debe ser cuidadosamente interpretada, ya que lo más probable es que éstas, en vez de constituir regeneración reciente, correspondan a individuos de edades similares o levemente inferiores a la edad media del renoval, que han quedado suprimidos. Este es un fenómeno corriente para especies de tolerancia media o intolerantes a la sombra (Oliver y Larson 1996), como D. winteri (Donoso 1993, Figueroa y Lusk 2001, Aravena et al. 2002, Gutiérrez et al. 2004). Por ejemplo, las distribuciones diamétricas reportadas por Navarro et al. (1999) para renovales de $D$. winteri de la costa de Valdivia son del tipo $\mathbf{J}$ inversa, con una enorme cantidad de individuos en las clases inferiores, a pesar de tratarse de renovales coetáneos. En renovales de menor tamaño hay mayor intercepción de luz en los doseles inferiores y además se manifiestan más las condiciones de drenaje imperfecto de los suelos Nadis; la habilidad competitiva de $D$. winteri en estas condiciones es mayor (Donoso 1993, Weinberger 1997) y, por lo tanto, aumentan las posibilidades de sobrevivencia de plantas establecidas en los orígenes del renoval.

Laureliopsis philippiana y las mirtáceas corresponden a especies muy tolerantes a la sombra (Donoso 1993, Weinberger 1997, Figueroa y Lusk 2001, Gutiérrez et al. 2004), pero la primera es más exigente en cuanto a condiciones de mejor drenaje del suelo (Weinberger 1997). Por ello se observan altas densidades de mirtáceas en todas las categorías de tamaño de renovales, pero no así en el caso de L. philippiana. Si bien es muy probable que esta especie sea capaz de regenerar bajo las condiciones de escasa luminosidad en el piso de renovales de $D$. winteri, en renovales de menor tamaño las malas condiciones de drenaje del suelo deberían constituir la principal limitante para el establecimiento de L. philippiana (Donoso et al. 1984, 1985; Weinberger 1997). En renovales de mayor tamaño, de grandes áreas basales y volúmenes (Navarro et al. 1999), con suelos menos húmedos que en estadios sucesionales iniciales, las condiciones de poca luminosidad en el sotobosque probablemente son muy similares a las iniciales, pero las mejores condiciones de drenaje del suelo permitirían el establecimiento de la regeneración de L. philippiana.

El comportamiento observado en E. cordifolia es de más difícil interpretación. Sin embargo, por tratarse de una especie semitolerante (Donoso et al. 1999), pero que requiere también de suelos bien drenados (Holdgate 1961, Donoso et al. 1984, 1985, Weinberger 1997), es probable que su mayor regeneración (a nivel de plántulas), observada en renovales de alturas intermedias, se explique porque en esta condición la especie encuentre un balance adecuado entre niveles de luminosidad apropiados para su regeneración y condiciones de mejor drenaje causadas por el incremento de la biomasa (Donoso et al. 1985). La luminosidad puede generarse por procesos o pulsos de alta mortalidad de individuos en el dosel superior (sensu Oliver y Larson 1991, Palik y Pretziger 1993) asociados a una alta competencia que ocurriría cuando el rodal alcanza su máxima capacidad de carga de acuerdo al área basal (figura 4). En rodales de menor tamaño habría limitaciones de drenaje y en rodales de mayor tamaño, de mayor estratificación, los claros generados por mortalidad de árboles del dosel superior probablemente no son lo suficientemente grandes como para permitir entrada de luz necesaria al piso para la regeneración de E. Cordifolia. Curiosamente, esta posible causa no es respaldada por los datos de regeneración según DMC (figura 3).

Las mirtáceas presentan una disminución de la regeneración en las clases superiores de altura y diámetro, particularmente en relación a la abundancia de brinzales (figuras 2 y 3 ). Este resultado es probablemente un reflejo de que, a pesar de que las mirtáceas de estos bosques son en general muy tolerantes a la sombra, hay un umbral de tamaño (sobre los $19 \mathrm{~m}$ de altura o los $15 \mathrm{~cm}$ de diámetro medio) que aparentemente representa una máxima capacidad de carga (manifestada en área basal; figura 4), sobre la cual la regeneración de mayor tamaño (y seguramente los árboles también) comienza a reducir su densidad debido a una intensa competencia.

Modelos de regeneración según categoría de altura y diámetro. Los modelos aportados por el estudio facilitan la comprensión de la dinámica de la regeneración en renovales delgados (latizales en terminología forestal) de D. winteri (cuadro 5). El mejor modelador de la regene-

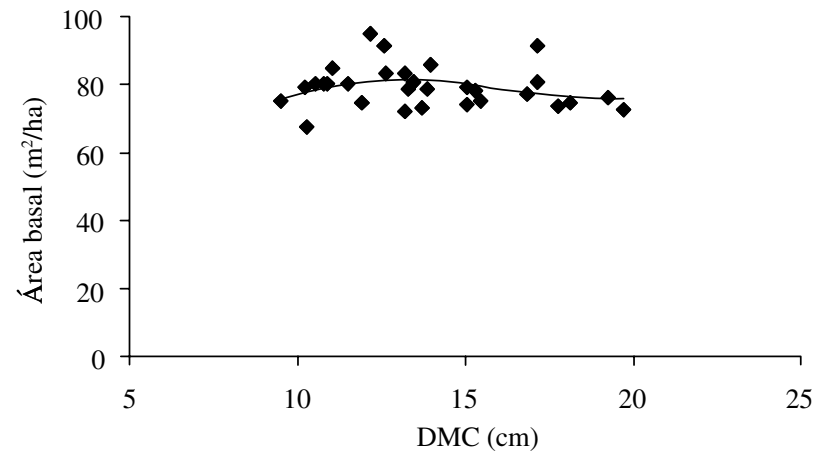

Figura 4. Relación del área basal y el diámetro medio cuadrático (DMC) en renovales de D. winteri del centro-norte de la isla de Chiloé con densidades relativas mayores a $80 \%$, la cual refleja un máximo de área basal alcanzado a un DMC aproximado de 13 a $15 \mathrm{~cm}$.

Relationship between basal area and quadratic stand diameter (DMC in Spanish) in D. winteri secondary forests in centralnorth Chiloé Island with relative densities $>80 \%$, reflecting a peak in basal area when DMC is between 13 and $15 \mathrm{~cm}$. 
ración para este estudio fue la AT y no el DMC, lo que sugiere que la estratificación del dosel arbóreo es relativamente más importante en cuanto a explicar la regeneración. Los modelos para $D$. winteri y E. cordifolia presentan un ajuste logarítmico negativo, tanto para la regeneración total como para los brinzales (cuadro 5), lo que indica que a medida que los renovales se desarrollan, las plántulas y brinzales de estas especies disminuyen exponencialmente (figuras 2 y 3 ). Por el contrario, $L$. philippiana mostró un ajuste potencial positivo para la regeneración (cuadro 5), y lineal para los brinzales, según DMC y $\mathrm{AT}$, consistente con el aumento de la regeneración y los brinzales en renovales más gruesos y altos (figuras 2 y 3). Finalmente, la no significancia de los ajustes para las mirtáceas es un reflejo de la gran plasticidad de éstas (Donoso 1993) y de la capacidad de regenerar en diferentes condiciones ambientales (Appel 1993, Figueroa y Hernández 2001, Figueroa y Lusk 2001). A pesar de estos resultados, es posible que una diferenciación entre las especies de mirtáceas refleje comportamientos diferenciados en la regeneración, como lo muestran los resultados de Weinberger (1997) y de Figueroa y Hernández (2001).

Interpretación sobre la dinámica regenerativa y aplicaciones para la silvicultura. Este estudio tuvo como hipótesis que $D$. winteri posee escasa o nula regeneración bajo su propio dosel en renovales de esta especie, independientemente de la estructura o la densidad, pero que la regeneración de especies más tolerantes, en particular L. Philippia$n a$, aumenta en estadios más avanzados de los renovales, es decir, cuando éstos tienen mayor altura y diámetro, y en condiciones de menos densidad. Estas hipótesis son respaldadas por los resultados, particularmente si se acepta la premisa de que las plántulas y brinzales de $D$. winteri observados en los distintos estadios de desarrollo no corresponden a regeneración recién establecida sino que a individuos suprimidos y establecidos al inicio de la sucesión. En todo caso, el estrecho rango de densidades relativas evaluadas no permite generar conclusiones con respecto al efecto de esta variable sobre la regeneración, aunque sí sugiere las tendencias hipotetizadas.

La composición de los bosques adultos en el centro norte de Chiloé corresponde a bosques dominados por pocos individuos de Nothofagus dombeyi (Mirb.) Oerst. (especie presente particularmente en suelos de pobre drenaje), D. winteri y E. cordifolia (generalmente en suelos mejor drenados) y abundantes individuos de L. philippiana y mirtáceas en los restantes estratos arbóreos (Donoso et al. 1984 y 1985, Armesto y Figueroa 1987, Armesto y Fuentes 1988, Aravena 1991, Armesto et al. 1995, Aravena et al. 2002, Gutiérrez et al. 2004). Esta composición es consistente con el escenario futuro que se prevé en los renovales del presente estudio, en el cual hay una alta competencia y gradual pérdida de densidad entre las especies del dosel superior, y cierta estabilidad (mirtá- ceas) o incremento en la abundancia de especies tolerantes a la sombra (L. philippiana). Este cambio puede ocurrir de acuerdo a la velocidad de la sucesión natural o puede ser acelerado o modelado parcialmente a través de silvicultura. Un renoval no manejado será un bosque futuro con una mayor importancia relativa de mirtáceas, L. philippiana y otras especies tolerantes, como podocarpáceas (Aravena et al. 2002). En cambio, uno manejado con un fuerte énfasis en aumentar la importancia relativa de especies comerciales, podría privilegiar los raleos a L. philippiana por sobre las mirtáceas, y generar espacios y oportunidades para favorecer el desarrollo, e incluso la regeneración, de E. cordifolia.

En futuros estudios deberían investigarse rodales con densidades relativas menores a $60 \%$, de modo de conocer los efectos de éstas sobre la regeneración, y por lo tanto predecir los potenciales efectos del manejo en estos renovales, de modo similar a lo desarrollado por Appel (1993) para renovales en la ladera occidental de la Cordillera de la Costa de Valdivia.

\section{AGRADECIMIENTOS}

Los autores agradecen al Proyecto DID S 2315 y al Convenio "Ecología y Silvicultura de los Bosques Nativos" (CONAF-UACH) de la Universidad Austral de Chile, y a dos revisores anónimos.

\section{REFERENCIAS}

Appel I. 1993. Evaluación de la regeneración en renovales de canelo (Drimys winteri) sometidos a diferentes niveles de intervención, en la Cordillera de la Costa, Provincia de Valdivia. Tesis Ingeniero Forestal. Facultad de Ciencias Forestales, Universidad Austral de Chile, Valdivia, Chile. $78 \mathrm{p}$.

Aravena JC. 1991. Análisis de la estructura y la flora de los bosques de Chiloé continental e insular. Tesis de Magíster en Ciencias. Santiago, Chile. Facultad de Ciencias, Universidad de Chile.

Aravena JC, M Carmona, C Pérez, JJ Armesto. 2002. Changes in tree species richness, stand structure and soil properties in a successional chronosequence in northern Chiloé Island, Chile. Revista Chilena de Historia Natural 75: 339-360.

Armesto JJ, J Figueroa. 1987. Stand structure and dynamics in the temperate rain forest of Chiloé Archipelago. Journal of Biogeography 14: 151-159.

Armesto JJ, E Fuentes. 1988. Tree species regeneration in a mid-elevation, temperate rainforest in Chiloé Island, Chile. Vegetatio 74: 151-159.

Armesto JJ, JC Aravena, C Villagrán, C Pérez, G Parker. 1996. Bosques templados de la Cordillera de la Costa. In Armesto J, C Villagrán, MK Arroyo eds. Ecología de los bosques nativos de Chile. Santiago, Chile. Universitaria. p. 199-213. 
Carmona MR, JC Aravena, CA Pérez, JJ Armesto. 2002. Coarse-woody debris in successional an primary temperate forest in Chiloé Island, Chile. Forest Ecology and Management 164: 265-275.

Corvalán P, L Araya, R Calquín, V Loewe, S Nieburh. 1987. El canelo: una alternativa de desarrollo para la Décima Región. Volumen IV Resultados. Universidad de Chile, Facultad de Ciencias Agrarias y Forestales, Departamento de Manejo de Recursos Forestales. 125 p.

Curtis RO. 1970. Stand density measures: an interpretation. Forest Science 16: 403-414.

Denton GH, TV Lowell, CJ Heusser, C Schluchter, BG Andersen, LE Heusser. 1999. Geomorphology, stratigraphy, and radiocarbon chronology of Llanquihue drift in the area of the Southern Lake District, Seno de Reloncaví and Isla de Chiloé, Chile. Geographyrafiska annaler 81(A): 167-229.

Di Castri F, ER Hayek. 1976. Bioclimatología de Chile. Pontificia Universidad Católica de Chile, Santiago, Chile. 128 p.

Donoso C, R Grez, B Escobar, P Real. 1984. Estructura y dinámica de bosques del tipo forestal siempreverde en el sector de Chiloé insular. Bosque 5(2): 82-104.

Donoso C, B Escobar, J Urrutia. 1985. Estructura y estrategias regenerativas de un bosque virgen de Ulmo (Eucryphia cordifolia Cav.)-Tepa (Laurelia philippiana Phil.) Looser en Chiloé, Chile. Revista Chilena de Historia Natural 58: 171-186.

Donoso C. 1993. Estructura, Variación y Dinámica de Bosques Templados de Chile y Argentina. Ecología Forestal. Santiago, Chile. Universitaria. 484 p.

Donoso C, PJ Donoso, ME González, V Sandoval. 1998. Los Bosques Siempreverdes. In Donoso C, A Lara eds. Silvicultura de los Bosques Nativos de Chile. Santiago, Chile. Universitaria. p. 261-289.

Donoso PJ, RD Nyland. 2005. Seedling density according to structure, dominance and understorey cover in old-growth forests stands of the evergreen forest type in the coastal range of Chile. Revista Chilena de Historia Natural 78: 51-63.

Donoso PJ, DN Soto, RA Bertin. 2007. Size-density relationships in Drimys winteri secondary forests of the Chiloe Island, Chile: Effects of physiography and species composition. Forest Ecology and Management. In press.

Drew TJ, JW Flewelling. 1977. Some recent Japanese theories of yield-density relationships and their applications to Monterrey Pine Plantations. Forest Science 23(4): 517-534.

FAO-UNESCO. 1971. Soil map of the world. South America, IV, Paris, Francia. 193 p.

Figueroa JA, CH Lusk. 2001. Germination requirements and seedling shade tolerance are not correlated in Chilean temperate rain forest. New Phytologist 152: 483-489.

Figueroa JA, JF Hernández. 2001. Seed germination responses in a temperate rain forest of Chiloé, Chile: effects of a gap and tree canopy. Ecología Austral 11: 37-45.

Gutiérrez AG, JJ Armesto, JC Aravena. 2004. Disturbance and regeneration dynamics of an old-growth North Patagonian rain forest in Chiloé Island, Chile. Journal of Ecology 92: 598-608.

Grez R, A Carmona. 1982. Utilización silvoagropecuaria de los terrenos Nadis; Caracterización de los suelos. Informe convenio. Universidad Austral de Chile, Valdivia, Chile. 24 p.

Holdgate MW. 1961. Vegetation and soils in south Chilean islands. Journal of Ecology 49 (3): 549-580.
Hunter ML. 1999. Maintainning Biodiversity in Forest Ecosystems. New York, USA. Cambridge University Press. 698 p.

Kozlowski TT. 2002. Physiological ecology of natural regeneration of harvested and disturbed forest stands: implications for forest management. Forest Ecology and Management 158(1): 195-221.

Loewe V. 1987. Evaluación de la regeneración natural del canelo (Drimys winteri) en la X Región. Tesis de Ingeniero Forestal. Santiago, Chile. Facultad de Ciencias Forestales, Universidad de Chile. 205 p.

Long JN, TJ Dean, SD Roberts. 2004. Linkages between silviculture and ecology: examination of several important conceptual models. Forest Ecology and Management 200: 249-261.

Navarro C, C Donoso, V Sandoval. 1999. Los renovales de canelo. In Donoso C, A Lara eds. Silvicultura de los Bosques Nativos de Chile. Santiago, Chile. Universitaria. p. 341-377.

Nyland RD. 2002. Silviculture concepts and applications. New York, USA. McGraw-Hill. 631 p.

Oliver CD. 1981. Forest development in North America following major disturbances. Forest Ecology and Management 3: 153-168.

Oliver CD, BC Larson. 1996. Forest Stand Dynamics. New York, USA. McGraw-Hill. 520 p.

Palik BJ, KS Pretziger. 1993 The repeatability of stem exclusion during even-aged development of bigtooth aspen dominated forests. Canadian Journal of Forest Research 23: 1156-1168.

Puettmann KJ, DW Hann, DE Hibbs. 1993. Evaluation of the size-density relationships for pure red alder and Douglas fir stands. Forest Science 39 (1): 7-27.

Puettmann KJ, DE Hibbs, DW Hann. 1992. The dynamics of mixed stands of Alnus rubra and Pseudotsuga menziessii: Extension of size-density analysis to mixture. Journal of Ecology 80(3) 449-458.

Reineke LH. 1933. Perfecting a stand density index for evenaged forests. Journal of Agricultural Research 46(7): 627638.

Rodríguez S. 1998. Antecedentes tecnológicos de Canelo (Drimys winteri Forst.). Bosque 19(1): 91-99.

Saldaña A, CH Lusk. 2003. Influencia de las especies del dosel en la disponibilidad de recursos y la regeneración avanzada en un bosque templados lluvioso del sur de Chile. Revista Chilena de Historia Natural 76: 639-650.

Smith DM, BR Larson, MJ Kelty, MS Ashton. 1997. The Practice of Silviculture: Applied Forest Ecology. New York, USA. John Wiley and Sons, Inc. 537 p.

Sokal RR, FJ Rohlf. 1995. Biometry: The principles and practice of statistics in biological research. New York, USA, Freeman and Co. 887 p.

Stokes M, T Smiley. 1968. An Introduction to Tree Ring Dating. Chicago, USA. University of Chicago Press.

Weinberger P. 1997. Definición de grupos ecológicos en formaciones boscosas siempreverdes de la zona austral de Chile. Bosque 18(2): 29-41.

Zeide B. 1995. A relationship between size of trees and their number. Forest Ecology and Management 72: 265-272.

Recibido: 13.03 .06

Aceptado: 12.10 .06 